\title{
A Review of the Distance and Structure of the Large Magellanic Cloud
}

\author{
David R. Alves \\ Columbia Astrophysics Lab., 550 W. 120th St., New York, NY, USA
}

The debate about the distance to the Large Magellanic Cloud (LMC) has an epic history full of controversial and dramatic claims (i.e., see review by Walker 2003), and yet in recent years a standard distance modulus has emerged due primarily to the completion of the Hubble Space Telescope (HST) Key Project to measure the Hubble constant (Freedman et al. 2001). The adopted standard distance modulus, $\mu_{0}=18.5 \pm 0.1 \mathrm{mag}$, yields $H_{0}=71 \pm 10 \mathrm{~km} \mathrm{~s}^{-1} \mathrm{Mpc}^{-1}$ (total error) in excellent agreement with that derived from the Wilkinson Microwave Anisotropy Probe: $H_{0}=72 \pm 5 \mathrm{~km} \mathrm{~s}^{-1} \mathrm{Mpc}^{-1}$ (Spergel et al. 2003), which lends considerable support to its accuracy.

The average of 14 recent measurements of the distance to the LMC implies a true modulus of $18.50 \pm 0.02 \mathrm{mag}$, and demonstrates a trend in the past 2 years of convergence toward a standard value. Table 1 is a summary of the distance results by method. Where both random $(r)$ and systematic $(s)$ errors are given, I adopt their arithmetic sum as the total error, and I use this to weight the average. I exclude one result, and two are assigned new error bars as noted in parenthesis. Last, I adopt the average distance modulus for the 4 eclipsing binary estimates as one result. Note that the eclipsing binaries yield a consistent mean distance, but their scatter is twice as large as their average measurement error. The reduced chi-squared is about 0.3 for the final average, and thus the adopted error bars are probably too conservative (the average uncertainty is $\pm 0.08 \mathrm{mag})$.

The recent results for the LMC distance demonstrate a remarkably high level of consistency. The possibility that a consensus on the LMC distance has been reached seems much more plausible now than it did at the conclusion of the HST Key Project only two years ago. A great American sports journalist once said famously, "The opera ain't over till the fat lady sings," to make a point that the outcome of a series of games was not yet determined. Regarding the convergence of published LMC distance results, I suggest to you that the fat lady has begun to sing.

There is no standard model for LMC structure, at least not yet, but in a series of recent papers a new understanding has emerged (van der Marel et al. 2002). It has been established that the photometric major axis of the LMC disk is about $50^{\circ}$ away from the line-of-nodes (along which the disk is at a constant distance equal to the center-of-mass distance), and thus the LMC is intrinsically elongated. Several independent estimates for the center of the LMC are consistent with the optical center; the exception is the center derived from the velocity field of $\mathrm{H} I$ gas. The velocity field of carbon stars and simple equilibrium models indicate that the LMC disk is thick like the Galactic thick 
Table 1. LMC distance moduli in the past 2 years

\begin{tabular}{llll}
\hline \hline Method & \multicolumn{1}{c}{$\mu_{0}$} & Method & \multicolumn{1}{c}{$\mu_{0}$} \\
\hline Red Clump & $18.493 \pm 0.033_{r} \pm 0.03_{s}$ & Mira & $18.48 \pm 0.08$ \\
Red Clump & $18.471 \pm 0.008_{r} \pm 0.045_{s}$ & Main Sequence (excl.) & $18.5-18.7$ \\
Red Clump & $18.54 \pm 0.10$ & Main Sequence & $18.58 \pm 0.08$ \\
Cepheid & $18.48( \pm 0.1)$ & SN 1987A & $18.46 \pm 0.12$ \\
Cepheid & $18.55 \pm 0.02_{r}( \pm 0.1)$ & EB (HV 982) & $18.51 \pm 0.05$ \\
Cepheid & $18.54 \pm 0.29$ & EB (EROS 1044) & $18.38 \pm 0.08$ \\
RR Lyrae & $18.50 \pm 0.07$ & EB (HV 5936) & $18.18 \pm 0.09$ \\
RR Lyrae & $18.43 \pm 0.06_{r} \pm 0.16_{s}$ & EB (HV 982) & $18.63 \pm 0.08$ \\
RR Lyrae & $18.55 \pm 0.07$ & Eclipsing binaries (ave.) & $18.46 \pm 0.08$ \\
RR Lyrae & $18.48 \pm 0.08$ & $\mathbf{1 8 . 5 0 \pm 0 . 0 2 ~ ( s . d . = 0 . 0 4 )}$ \\
\hline \multicolumn{5}{r}{ Wtd. ave. } \\
\hline \hline
\end{tabular}

disk and flared. The vertical exponential scale height of carbon stars attains a value of about $1 \mathrm{kpc}$ at locations 5-6 degrees from center, and is only about 100 pc in the central bar region.

The LMC is the nearest example besides the Milky Way of a disk galaxy that has a stellar halo (Minniti et al. 2003). Although the LMC has a halo, it does not have a bulge, and in this way it is similar to M33. It not known whether or not the Population II distance indicators like RR Lyrae variables are distributed in the same way as the young and intermediate-age stellar populations which represent the bulk of the LMC disk, and hence whether or not geometric inclination-corrections appropriate for the disk should apply. However, the kinematics of the halo and disk stars in the LMC are distinct.

Finally, I note that the warps in the LMC disk recently discovered using the color and magnitude of the red clump (Olsen \& Salyk 2002) are suspiciously found in regions of high reddening, and could be caused by a change in the mix of young and old red clump stars which would bias the reddening correction. Old and intrinsically redder red clump stars, if they exist, could be distributed in a different manner than the young (disk) clump stars, and thus it is possible for the population mix of the clump to vary in unexpected ways across the LMC.

\section{References}

Freedman, W. L., et al. 2001, ApJ, 553, 47

Minniti, D., Borissova, J., Rejkuba, M., Alves, D. R., Cook, K. H., \& Freeman, K. C. 2003, Science, 301, 1508

Olsen, K. A. G., \& Salyk, C. 2002, AJ, 124, 2045

Spergel, D. N., et al. 2003, ApJS, 148, 175

van der Marel, R. P., Alves, D. R., Hardy, E., \& Suntzeff, N. B. 2002, AJ, 124, 2524

Walker, A. R. 2003, in "Stellar Candles for the Extragalactic Distance Scale," Lecture Notes in Physics (Springer), eds. D. Alloin and W. Gieren (astro-ph/0303011) 